\title{
Neural Mode Jump Monte Carlo
}

\author{
Luigi Sbailò* and Manuel Dibak* \\ Freie Universität Berlin, Department of Mathematics and Computer Science, Arnimallee 6, 14195 \\ Berlin, Germany \\ Frank Noé \\ FRANK.NOE@FU-BERLIN.DE \\ Freie Universität Berlin, Department of Mathematics and Computer Science, Arnimallee 6, 14195 \\ Berlin, Germany \\ Freie Universität Berlin, Department of Physics, Arnimallee 6, 14195 Berlin, Germany \\ Rice University, Department of Chemistry, Houston TX, 77005, United States
}

\begin{abstract}
Markov chain Monte Carlo methods are a powerful tool for sampling equilibrium configurations in complex systems. One problem these methods often face is slow convergence over large energy barriers. In this work, we propose a novel method which increases convergence in systems composed of many metastable states. This method aims to connect metastable regions directly using generative neural networks in order to propose new configurations in the Markov chain and optimizes the acceptance probability of large jumps between modes in configuration space. We provide a comprehensive theory and demonstrate the method on example systems.

Keywords: efficient MCMC, high dimensional distribution, invertible models, metastable states
\end{abstract}

\section{Introduction}

Markov chain Monte Carlo (MCMC) methods are used to sample the equilibrium distribution of systems whose probability distribution is otherwise analytically intractable. An efficient MCMC generator proposes moves that quickly decorrelate the samples while having a large acceptance probability. A common choice are moves with a random displacement in configuration space Metropolis et al. (1953). As complex systems at equilibrium visit only a small fraction of the whole configuration space, these random displacements have to be very small to be accepted. However small moves are only efficient at sampling local conformations of the energy landscape, while crossing large energy barriers requires a multitude of sampling steps. This problem is particularly evident when the system is composed of many metastable states, where it is often computationally infeasible to cross energy barriers multiple times. This leads to a problem known as broken ergodicity, or quasi-ergodicity, which implies that the probability to cross a energy barrier is so low that simulations converge too slow to be practical.

In the last decades, many different methods have been developed to circumvent this problem: One class of methods varies the temperature during the sampling process as the crossing time over energy barriers exponentially decreases with inverse temperatures. The

* equal contribution 
two most widely recognized methods in this class are simulated Marinari and Parisi (1992); Geyer and Thompson (1995) and parallel tempering Geyer (1991); Hukushima and Nemoto (1996b) which operate on fixed set of temperatures. Simulated tempering randomly changes the temperature of the sampler from a set of discrete temperatures while remaining at equilibrium in an augmented temperature-configuration space. In parallel tempering multiple simulations at different temperatures are carried out in parallel and samples are randomly exchanged between the different temperatures. These methods rely on a significant overlap of the energetic distributions at different temperatures, therefore the temperature range has to be chosen carefully.

A different class of methods biases the potential landscape in order to enable transitions over energy barriers and recovers the unbiased distribution by re-weighting. Metadynamics Laio and Parrinello (2002) does this in an iterative fashion, where the bias potential is increased in areas where the system resides a long time thus pushing the system out of metastable states. Recent development suggest the usage of deep learning to find an optimal bias potential Zhang et al. (2019). Umbrella sampling Torrie and Valleau (1977) runs several sampling iterations with bias potentials placed along a pre-defined coordinate and thus pushes the system from one end to the other of this reaction coordinate.

A novel method are Boltzmann Generators Noé et al. (2019), which uses deep learning in order to learn to draw unbiased samples from a target distribution $p_{X}(\mathbf{x}) \propto \exp (-u(\mathbf{x}))$ by combining an exact probability generator such as a normalizing Flow Rezende and Mohamed (2015); Dinh et al. (2016) with reweighting Noé et al. (2019); Albergo et al. (2019); Nicoli et al. (2019).

Another recently developed approach Stern (2007); Nilmeier et al. (2011a); Chen and Roux (2015) constructs reversible moves between equilibrium states as a collection of small out-of-equilbrium trajectories. This approach also depends on the path connecting the equilibrium states, and a system specific protocol to generate the candidate state must be designed.

Smart Darting Monte Carlo Andricioaei et al. (2001); Roberts et al. (2012) is a promising method that alternates local and long range moves from one region of the configuration space to another that is arbitrary far. These moves are attempted between small spheres around local minima. In high dimensions however the fraction of the spheres to the total volume becomes vanishingly small and therefore finding a sphere by random exploration becomes very unlikely. This problem is circumvented in ConfJump Walter and Weber (2006) by finding the closest energy minimum, and attempting long range moves by translation to another energy minimum.

The generation of long range moves is challenging when the energy landscape is rough, since the potential energy surface in the region surrounding local minima can drastically change between the different minima. In this case, using trivial translation as long range moves would most likely cause large energy differences and is likely to be rejected. Instead, a specific bijective function pairing points in order to keep the energy difference small needs to be employed. However, constructing such bijection manually would require detailed knowledge of the system and is practically impossible in multi-dimensional systems.

Recent advances in the field of machine learning have permitted to deal with problems that were not solvable with a sole human understanding, and, more specifically, deep neural networks (DNNs) are an ideal tool to facilitate the construction of a bijective function. 
DNNs have already been employed to construct MCMC moves. Current methods use DNNs to approximate the distribution and thus speeding up sampling Shen et al. (2018), projecting onto high probability manifolds Habib and Barber (2019) or use a latent space representation in order to propose moves Noé et al. (2019); Albergo et al. (2019).

Two recent methods are using reversible network architectures in order to improve Hamiltonian Monte Carlo (HMC): Song et al. (2017) propose steps by applying a volume preserving flow to the augmented configuration space. Levy et al. (2018) augment the leapfrog algorithm commonly used in HMC with DNNs and thus alter the classical path of the system while relying on forces. Both are trained for sampling efficiency in a unsupervised fashion and therefore rely on random exploration of configuration space in order to find metastable states.

In this paper, we present neural mode jump Monte Carlo (Neural MJMC), a novel method to efficiently sample the equilibrium distribution of complex many-body systems with unbiased Markov chains. In this scheme neural networks are trained to propose "neural" moves that directly connect different metastable states. These proposals do not try to approximate a classical path between start and endpoint which gives them the freedom to connect regions in phase space that are arbitrarily far apart. The method requires a prior knowledge of the position of the metastable states in configuration space, which could e.g. be obtained from x-ray scattering experiments. Local displacements and neural moves are randomly alternated in a combined scheme to accelerate the convergence rate of Markov chains. Configurations from different metastable states are used to train the networks, which are optimized to produce high acceptance probability moves. Local exploration ensures ergodicity of the scheme, while neural moves accelerate convergence to equilibrium, realizing an accurate and deep exploration of the configuration space.

\section{Theory}

A sufficient condition to ensure that a Markov chain asymptotically samples the equilibrium distribution is ergodicity and detailed balance. Given the system in a configuration $\mathbf{x}$ a new state $\mathbf{y}$ is added to the chain with a transition probability $p(\mathbf{x} \rightarrow \mathbf{y})$. The transition probability is defined to satisfy the condition of detailed balance

$$
\pi(\mathbf{x}) p(\mathbf{x} \rightarrow \mathbf{y})=\pi(\mathbf{y}) p(\mathbf{y} \rightarrow \mathbf{x}),
$$

where $\pi(\mathbf{x})$ is the equilibrium distribution. In the Metropolis-Hastings algorithm Metropolis et al. (1953); Hastings (1970), the transition probability is decomposed in two logical steps: firstly, a new configuration $\mathbf{y}$ is drawn from a proposal density $p_{\text {prop }}(\mathbf{x} \rightarrow \mathbf{y})$, then the new state is accepted with an acceptance probability $p_{\text {acc }}(\mathbf{x} \rightarrow \mathbf{y})$. If the transition is accepted, the new state $\mathbf{y}$ is added to the Markov chain, otherwise the previous state $\mathbf{x}$ is added to the Markov chain.

In Neural MJMC, additionally the proposal probability is split into two steps: firstly, a proposal density is selected from a pre-defined list of proposal densities on the current state $\mathbf{x}$, then a new state $\mathbf{y}$ is drawn from the extracted proposal density. Proposal densities are distinguished between local proposals and neural proposals, where local proposals generate local moves through random displacement, as already proposed in the Metropolis scheme, and neural proposals connect different metastable states. 
Let us assume that the configuration space $\Omega$ is decomposed into a number of non overlapping subsets called cores $\left\{\Omega_{\alpha}\right\}_{\alpha \leq N} \subset \Omega$, with $\cup_{\alpha} \Omega_{\alpha}=\Omega$, each representing one of the $N$ metastable states. We define the neural proposal $K_{\alpha \beta}$, as the density that proposes transitions from the core $\Omega_{\alpha}$ to the core $\Omega_{\beta}$. Assuming the system in the state $\mathbf{x} \in \Omega_{\alpha}$, the probability to extract the neural proposal $K_{\alpha \beta}$ is $p_{\alpha \beta}(\mathbf{x})$. Once $K_{\alpha \beta}$ has been selected, a state $\mathbf{y} \in \Omega_{\beta}$ is drawn from the selection probability $p_{\text {prop }}^{\alpha \beta}(\mathbf{x} \rightarrow \mathbf{y})$.

A neural proposal $K_{\alpha \beta}$ can only be selected within the core $\Omega_{\alpha}$ and with constant probability $p_{\alpha \beta}(\mathbf{x})=p_{\alpha \beta} \chi_{\Omega_{\alpha}}(\mathbf{x})$, where $\chi_{\Omega}(\mathbf{x})$ denotes the characteristic function. We assume that each pair of states $(\alpha, \beta)$ is only connected by one neural proposal $K_{\alpha \beta}$ and that there exists an inverse proposal $K_{\beta \alpha}$ connecting $\beta$ with $\alpha$. Under these assumptions, a proposed move starting in $\Omega_{\alpha}$ with selected neural proposal $K_{\alpha \beta}$ fulfills detailed balance if it is accepted with probability

$$
p_{a c c}^{\alpha \beta}(\mathbf{x} \rightarrow \mathbf{y})=\min \left\{1, \frac{\pi(\mathbf{y}) p_{\beta \alpha} p_{p r o p}^{\beta \alpha}(\mathbf{y} \rightarrow \mathbf{x})}{\pi(\mathbf{x}) p_{\alpha \beta} p_{\text {prop }}^{\alpha \beta}(\mathbf{x} \rightarrow \mathbf{y})}\right\} .
$$

We parameterize the neural proposal $K_{\alpha \beta}$ and its inverse $K_{\beta \alpha}$ connecting the cores $\Omega_{\alpha}$ and $\Omega_{\beta}$, as a bijective function $\mu_{\alpha \beta}(\cdot)$ pairing the states defined in the two cores, i.e $\mathbf{y}=\mu_{\alpha \beta}(\mathbf{x})$, $\mu_{\alpha \beta}^{-1}(\mathbf{y})=\mathbf{x}, \forall \mathbf{x} \in \Omega_{\alpha}$, where $\mathbf{x} \in \Omega_{\alpha}, \mathbf{y} \in \Omega_{\beta}$. Thus for each pair of different cores $\left(\Omega_{\alpha}, \Omega_{\beta}\right)$ a bijective function $\mu_{\alpha \beta}(\cdot)$ is defined. The probability distribution of neural proposals is then represented with Dirac delta distributions and the acceptance specifies to

$$
p_{a c c}^{\alpha \beta}(\mathbf{x} \rightarrow \mathbf{y})=\min \left\{1, \frac{\pi(\mathbf{y}) p_{\alpha \beta} \delta\left(\mathbf{x}-\mu_{\alpha \beta}^{-1}(\mathbf{y})\right)}{\pi(\mathbf{x}) p_{\beta \alpha} \delta\left(\mathbf{y}-\mu_{\alpha \beta}(\mathbf{x})\right)}\right\} .
$$

Using the change of variable formula in the Dirac distribution $\delta\left(\mathbf{x}-\mu_{\alpha \beta}^{-1}(\mathbf{y})\right)=\left|\operatorname{det} J\left(\mu_{\alpha \beta}(\mathbf{x})\right)\right| \delta(\mathbf{y}-$ $\left.\mu_{\alpha \beta}(\mathbf{x})\right)$, the acceptance probability for neural moves can be simplified to

$$
p_{a c c}^{\alpha \beta}(\mathbf{x} \rightarrow \mathbf{y})=\min \left\{1, \frac{\pi(\mathbf{y}) p_{\alpha \beta}}{\pi(\mathbf{x}) p_{\beta \alpha}}\left|\operatorname{det} J\left(\mu_{\alpha \beta}(\mathbf{x})\right)\right|\right\} .
$$

In case that the local proposal $(\alpha=\beta)$ is selected, the inverse move is only possible with another local proposal $K_{\alpha \alpha}$. Note that a local move may leave the current core and the proposal probability for the inverse move might change. Thus the acceptance probability for a local move reduces to

$$
p_{a c c}^{\alpha \alpha}(\mathbf{x} \rightarrow \mathbf{y})=\min \left\{1, \frac{\pi(\mathbf{y}) \sum_{\beta} \chi_{\Omega_{\beta}} p_{\beta \beta}}{\pi(\mathbf{x}) p_{\alpha \alpha}}\right\} .
$$

In order to ensure ergodicity there needs to be a finite probability of selecting the local proposal in all cores. In Algorithm 1, we summarize the Neural MJMC sampling scheme.

\subsection{Optimal proposal density}

In order to achieve fast decorrelation of the Markov chain the neural proposal functions $\mu_{\alpha \beta}$ should maximize the acceptance in both directions. This is quantified by maximizing the 


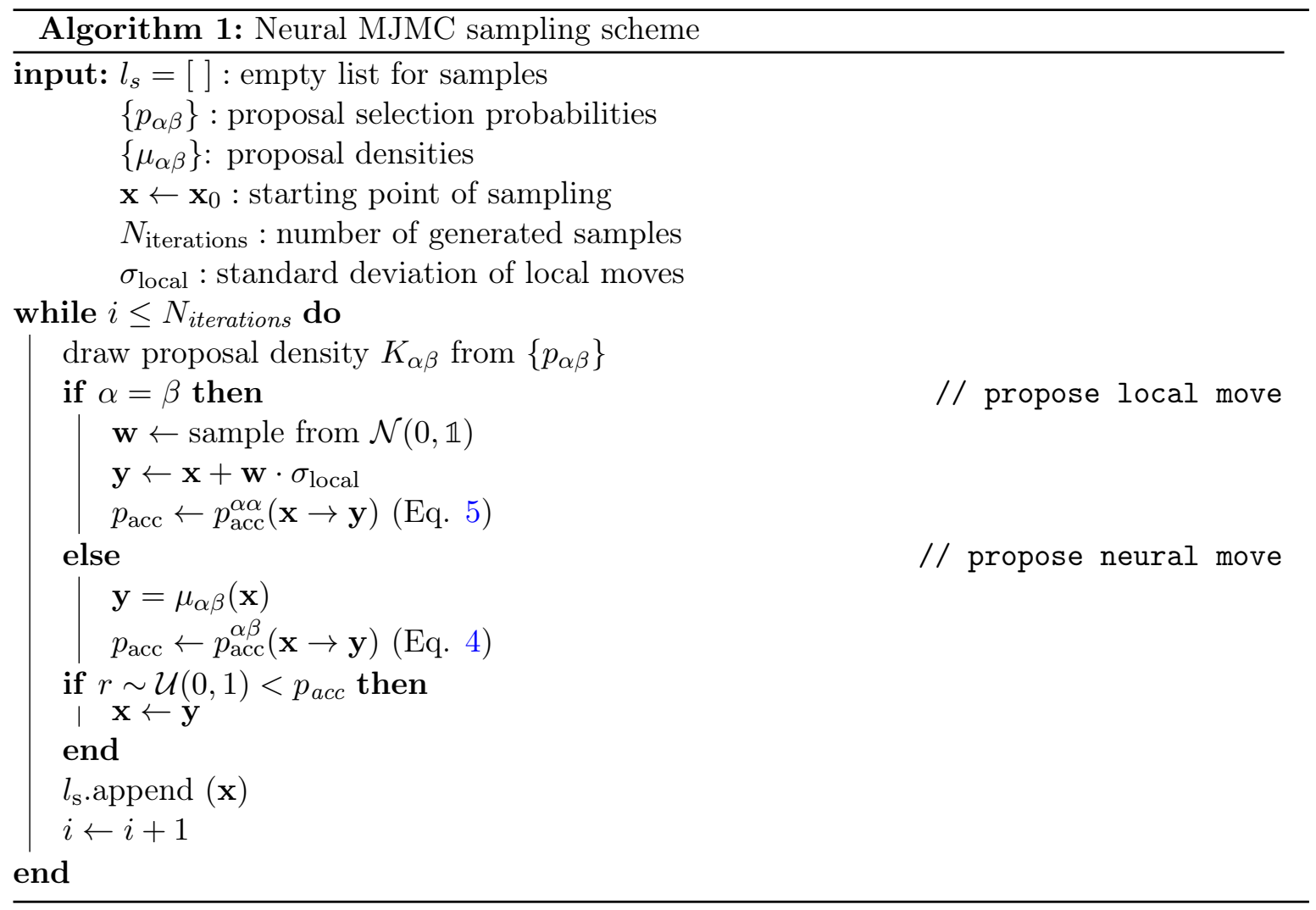


expected log probability that the moves proposed by $\mu_{\alpha \beta}$ are accepted in both directions. Using Jensen's inequality we find

$$
\begin{aligned}
& \max _{\mu_{\alpha \beta}} \log \mathbb{E}_{\mathbf{x} \sim \Omega_{\alpha}}\left[p_{\text {pacc }}(\mathbf{x} \rightarrow \mathbf{y}) p_{\text {pacc }}(\mathbf{y} \rightarrow \mathbf{x})\right] \geq \max \mathbb{E}\left\{\log \left[p_{\text {pacc }}(\mathbf{x} \rightarrow \mathbf{y}) p_{\text {pacc }}(\mathbf{y} \rightarrow \mathbf{x})\right]\right\} \\
= & \max _{\mu_{\alpha \beta}} \mathbb{E}[\min (0, \log f)+\min (0,-\log f)]=\max _{\mu_{\alpha \beta}} \mathbb{E}[\min (\log f,-\log f)]=\max _{\mu_{\alpha \beta}} \mathbb{E}[-|\log f|],
\end{aligned}
$$

where $f=\frac{\pi(\mathbf{y}) p_{\alpha \beta}}{\pi(\mathbf{x}) p_{\beta \alpha}}\left|\operatorname{det} J\left(\mu_{\alpha \beta}(\mathbf{x})\right)\right|$. Using the stationary distribution in the canonical ensemble $\pi(\mathbf{x}) \propto \exp (-\beta V(\mathbf{x}))$, with the thermal energy $\beta^{-1}=k_{B} T$, the potential energy $V(\mathbf{x})$ of the system under consideration and assuming that $\mu_{\alpha \beta}$ is a bijection between the cores $(\alpha, \beta)$, we can rewrite the equation above to find

$$
\min _{\mu_{\alpha \beta}} \mathbb{E}\left[\beta\left|\Delta V_{\alpha \beta}(\mathbf{x})+k_{B} T \log \right| \operatorname{det} J_{\mu_{\alpha \beta}}(x)\left|+\Delta R_{\alpha \beta}\right|\right],
$$

with the potential difference $\Delta V_{\alpha \beta}(\mathbf{x}):=V(\mathbf{x})-V\left(\mu_{\alpha \beta}(\mathbf{x})\right)$ and the log selection ratio $\Delta R_{\alpha \beta}:=-k_{B} T \log p_{\alpha \beta} / p_{\beta \alpha}$. Note that the term inside the modulus is equivalent to the Kulbach-Leibler divergence between the transformed distribution $\mu_{\alpha \beta}\left(\Omega_{\alpha}\right)$ and the target distribution $\Omega_{\beta}$ as found in Noé et al. (2019).

We can interpret this result in a physically meaningful manner by applying the triangular inequality $\mathbb{E}[-|\log f|] \geq-|\mathbb{E}[\log f]|$, identifying $\Delta S=-k_{B} \mathbb{E}\left[\log \left|\operatorname{det} J_{\mu_{\alpha \beta}}(x)\right|\right]$ as the change of entropy (see Appendix A for details) and $\Delta U=\mathbb{E}\left[\Delta V_{\alpha \beta}(\mathbf{x})\right]$ as the change of internal energy under the transformation $\mu_{\alpha \beta}(\mathbf{x})$. We observe that the expected log acceptance is lower bound by the absolute change in free energy $\Delta F=\Delta U-T \Delta S$ under the transformation $\mu_{i j}(\cdot)$ divided by thermal energy

$$
\mathbb{E}\left\{\log \left[p_{\text {pacc }}(\mathbf{x} \rightarrow \mathbf{y}) p_{\text {pacc }}(\mathbf{y} \rightarrow \mathbf{x})\right]\right\} \geq-\beta\left|\Delta F+\Delta R_{i j}\right| .
$$

This result shows that we can use the freedom in the proposal selection ratio in order to maximize the bi-directional acceptance.

\section{Neural network architecture}

As a neural moves relies on an exactly invertible function with a computationally feasible Jacobian, this must also be reflected in the choice of the neural network architecture. Recently, several such structures have been proposed Song et al. (2017); Dinh et al. (2016); Rezende and Mohamed (2015); Chen et al. (2018) and they vary in expressiveness and computational cost.

In order to ensure that outputs of the network $\mu_{\alpha \beta}(\cdot)$ are in the correct well, a harmonic bias potential centered in the target core is added during training

$$
V_{\text {bias }}(\mathbf{x})=\left\{\begin{array}{ll}
k\left(\mathbf{x}-\mathbf{x}_{\alpha}\right) & \mathbf{x} \in \Omega_{\alpha} \\
k\left(\mathbf{x}-\mathbf{x}_{\beta}\right) & \mathbf{x} \in \Omega_{\beta}
\end{array},\right.
$$

where $\mathbf{x}_{\alpha}$ is the reference configuration in core $\alpha$, resulting in the biased system $\tilde{V}(\mathbf{x})=$ $V(\mathbf{x})+V_{\text {bias }}(\mathbf{x})$ used during training. The network is trained in several stages, gradually 

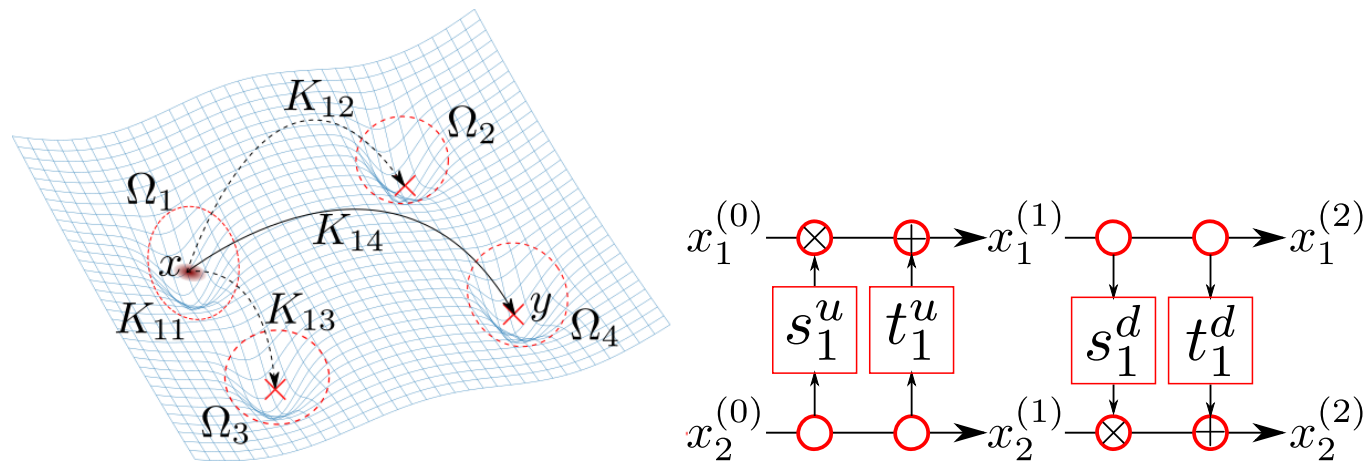

Figure 1: Left: Schematic figure of Neural MJMC scheme. Given configuration $\mathbf{x}$ in core $\Omega_{\alpha}$ there are three neural and one local proposals available, as denoted by arrows. One of these is selected and a new state $\mathbf{y}$ is proposed. Right: Architecture of the RNVP networks that are used as reversible networks for the examples in this paper. The input vector $\mathbf{x}$ is separated into two disjoint sets of coordinates $\mathbf{x}_{1}, \mathbf{x}_{2}$, and at each iteration one subset undergoes a nonlinear transformation and is multiplied and added to the other subset. The transformation can easily be inverted.

lowering the strength of the bias potential. To find the reference configurations $\mathbf{x}_{\alpha}$ k-means clustering is run on samples generated from local MCMC sampling in either well. Training sets of both of the wells are generated for a set of gradually decreasing bias strengths $\left\{k_{i}\right\}_{i \leq N_{k}}$. After convergence of the training at $k_{i}$, the training set is exchanged and training is restarted with $k_{i+1} \leq k_{i}$. This allows for a slowly expanding training set, which enables the network to learn how to generate meaningful moves on a gradually more complex set of training data. The loss that is to be minimized during training is given by the bi-directional acceptance 7

$$
C_{\mathrm{acc}}=\mathbb{E}_{\mathbf{x} \sim \Omega_{\alpha}}\left\{\left[\Delta \tilde{V}_{\alpha \beta}(\mathbf{x})+k_{B} T \log \left|\operatorname{det} J_{\mu_{\alpha \beta}}(\mathbf{x})\right|\right]^{2}\right\}
$$

where the square of the norm is used in order to penalize high energies more heavily. Training is performed in the forward and backward direction and the same loss applies to samples from core $\Omega_{\beta}$ with exchanged labels $\alpha \leftrightarrow \beta$.

\section{Numerical Experiments}

We demonstrate Neural MJMC on two examples: a two dimensional potential landscape with three minima and a system consisting of two dimer particles which are suspended in a bath of repulsive particles. The detailed training parameters are given in Appendix C.

As a good compromise between computational cost and expressiveness, we use real nonvolume preserving transformations (RNVP) Dinh et al. (2016) for these examples. In a $R N V P$ layer the configuration vector $\mathbf{x} \in \mathbb{R}^{N \times \operatorname{dim}}$ is split into two vectors $\mathbf{x}_{1}$ and $\mathbf{x}_{2}$. As we 


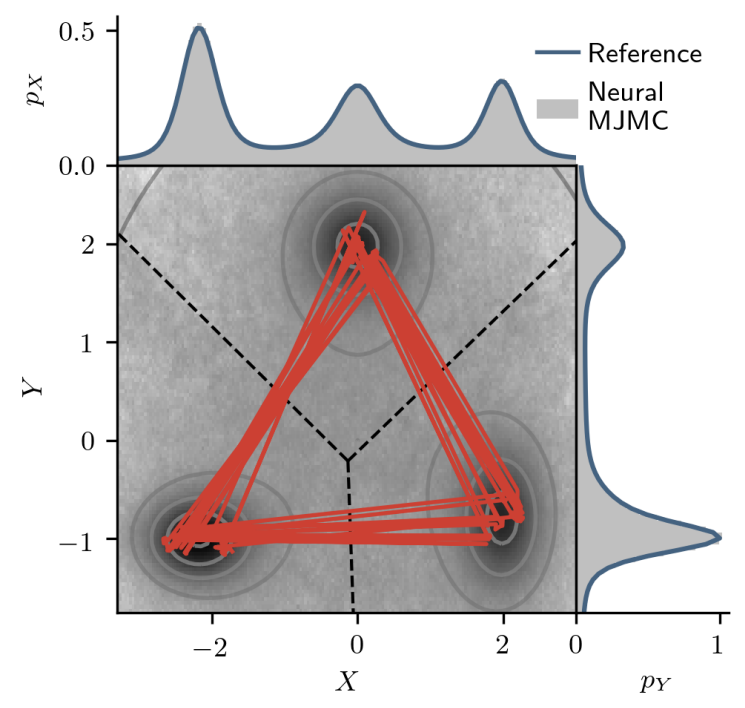

Figure 2: Two dimensional histogram (center) of samples from the 2D Gaussian triple well potential generated by Neural MJMC with a short section of the Markov chain (red solid line) and marginal distributions $p_{X}$ (top) and $p_{Y}$ (right). The black dashed line depicts the border between the states which are defined by a Voronoi tessellation. Convergence to the correct Boltzmann distribution can be observed from the histograms of the marginal distributions, where the blue line is the reference solution from numerical integration of the system's Boltzmann distribution.

deal with two dimensional systems, we split along the $x$ and $y$ coordinates of all particles such that $\mathbf{x}_{1}, \mathbf{x}_{2} \in \mathbb{R}^{N \times 1}$. One RNVP layer consists of two update steps in which the first subset is updated based on the second while the second is kept constant, and vice versa

$$
\begin{aligned}
{\left[\begin{array}{l}
\mathbf{x}_{1}^{(i)^{\prime}} \\
\mathbf{x}_{2}^{(i)^{\prime}}
\end{array}\right] } & =\left[\begin{array}{c}
\left.\mathbf{x}_{1}^{(i)} \odot \exp \left[S_{i}\left(\mathbf{x}_{2}^{(i)}\right)\right]+T_{i}\left(\mathbf{x}_{2}^{(i)}\right)\right] \\
\mathbf{x}_{2}^{(i)}
\end{array}\right] \\
{\left[\begin{array}{c}
\mathbf{x}_{1}^{(i+1)} \\
\mathbf{x}_{2}^{(i+1)}
\end{array}\right] } & =\left[\begin{array}{c}
\mathbf{x}_{\mathbf{1}}^{(i)^{\prime}} \\
\left.\mathbf{x}_{2}^{(i)^{\prime}} \odot \exp \left[S_{i}^{\prime}\left(\mathbf{x}_{1}^{(i)^{\prime}}\right)\right]+T_{i}^{\prime}\left(\mathbf{x}_{1}^{(i)^{\prime}}\right)\right]
\end{array}\right.
\end{aligned}
$$

where the $S_{i}, T_{i}, T_{i}^{\prime}, S_{i}^{\prime}$ are dense feed forward neural networks. The above system of equations represent one RNVP block, and arbitrary many of these blocks can be serially stacked resulting in more complex transformations (see Fig. 1). The logarithm of the Jacobian determinant of this transformation is given by the sum over all the outputs of all the scaling layers $\log \left|\operatorname{det} J_{\mu_{\alpha \beta}}(\mathbf{x})\right|=\sum_{i} \sum_{j}\left(S_{i j}+S_{i j}^{\prime}\right)$.

\subsection{Gaussian triple well}

As an example for a system with multiple states, we demonstrate Neural MJMC on a two dimensional potential landscape consisting of 3 Gaussian shaped wells. We define the 3 cores 



Figure 3: Left: Free energy along the distance between the dimer particles. The corresponding bands represent reference values obtained by umbrella sampling. The neural network has been trained at temperature $T=T_{0}$, then simulations at different temperatures have been performed using Neural MJMC. Simulations are run for $1.5 \times 10^{7}$ steps, and error bars are generated from several sampling runs. In this figure, we observe that Neural MJMC correctly samples the free energy along the reaction coordinate of the system at different temperatures. Right top: Reference configurations in the closed (left) and open (right) dimer configuration. The dimer particles are displayed in blue, and solvent particles in grey. The strongly repulsive potential does not allow for significant overlaps between particles at equilibrium. Right bottom: Dimer interaction potential $E_{d}$ as a function of the dimer distance. 


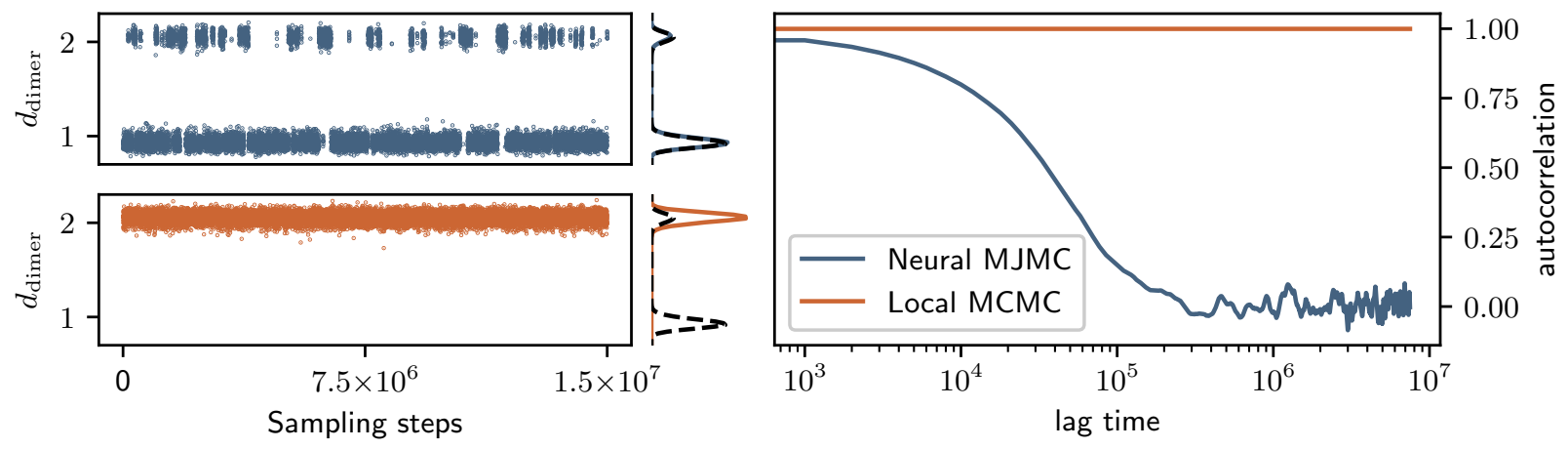

Figure 4: Left: Dimer distance over a single realization using Neural MJMC (top), and using local MCMC (bottom). Right) Histogram of the dimer distance obtained by the displayed trajectory, with the reference value displayed as the black dashed line. Spontaneous transitions with local MCMC are not observed at this time scale. Neural MJMC explores both metastable states in the trajectory multiple times and correctly reproduces the distribution of dimer distances. Right: $\mathrm{Au}-$ tocorrelation of the dimer distance. Neural moves allow for a fast exploration of both metastable states, accelerating the production of uncorrelated samples. In this figure, it is evident that Neural MJMC frequently generates uncorrelated samples, and short trajectories are sufficient to reconstruct the right distribution. In contrast, configurations generated with local MCMC are highly correlated, as they do not cross the energy barrier. 
by a Voronoi tessellation for which we use the minima of the Gaussians as centers. Each of the three neural proposals are trained independently on configurations sampled from the minima. In the sampling step, 100 independent trajectories of length $10^{5}$ steps are generated and averaged. We compare the marginal distributions $p_{X}$ and $p_{Y}$ which are the projections of the Boltzmann distribution on the $X$ and $Y$ axes and observe great agreement to results from numerical integration of the Boltzmann distribution (see Fig. 2).

\subsection{Dimer in repulsive Lennard Jones bath}

As a bigger challenge Neural MJMC is applied to a two-dimensional system composed of a bistable dimer immersed in a bath of strongly repelling particles and confined to a box. The bistable dimer potential has a minimum in the closed and open configurations which are separated by a high energy barrier (see Fig. 3 right bottom). Opening and closing of the dimer requires a concerted motion of the solvent particles, that makes it difficult to sample the physical path connecting the two configurations (see Ref. Noé et al. (2019) for a more detailed description of the system).

The open and closed configuration serve as cores (see Fig. 3 right top) in Neural MJMC and are distinguished by the distance between the dimer particles. The neural network is trained on states sampled independently in the closed and open configuration at four different bias strengths with $10^{5}$ samples for each well and bias. As the system is invariant under permutation of solvent and dimer particles, neural moves for each permutation of the system would have to be learned independently. This is clearly unfeasible, as the number of permutation scales factorial in the particle number. This is circumvented by permutation reduction, i.e. re-labeling the particles such that the distance to the reference configuration is minimized. This is realized using the Hungarian algorithm Kuhn (1955) with the reference configurations as target.

Each neural network in the RNVP architecture consists of three hidden layer with 76 nodes. The transformation consists of a total of 20 RNVP layers and contains approximately $1.4 \times 10^{6}$ trainable parameters. Neural MJMC is used to generate a single trajectory with $1.5 \times 10^{7}$ steps, where the probability of neural moves is set to $1 \%$. In terms of computational performance, sampling with Neural MJMC is approximately a factor of four slower than MCMC with local displacements for this system. This slow down arises from the evaluation of the network and the remapping of particles. As a reference value, we use umbrella sampling to sample the free energy along the dimer distance.

Neural moves cause direct transitions between the two metastable states and thus a rapid exploration of the configuration space. The convergence to the Boltzmann distribution is observed as shown in Fig. 3. An estimate for the crossing time with only local moves can be found to be at the order of $10^{12}$ sampling steps at $T=1$ from the Kramer's problem which make exhausting simulations unfeasible. In Neural MJMC many crossings of the energy barrier can be observed (Fig. 4 top). This is also reflected in the autocorrelation function where samples generated with local MCMC remain highly correlated, while it decays in Neural MJMC simulations on a scale of approximately $10^{5}$ sampling steps (see Fig. 4 bottom). Thus generating the desired uncorrelated samples of the equilibrium distribution. 


\section{Conclusion}

In this paper, we have presented Neural Mode Jump Monte Carlo, a novel method that allows for efficient sampling of Boltzmann distributions of complex systems composed of many metastable states. The method uses neural networks in order to parametrize bijections between metastable regions in phase space and optimizes these networks for bi-directional acceptance probability. By combining short steps given by random displacements and large jumps between metastable states, the method is able to converge quickly to the Boltzmann distribution. This is especially evident in systems where large potential barriers are providing obstacles to convergence of other methods. The method is demonstrated on two toy examples, one with several bijections in two dimensions and a high dimensional system consisting of a particle dimer in a bath of Lennard Jones particles.

The reversible neural network architecture used in this work is also used in the field of generative probabilistic modeling. Considering the great attention this field is lately facing, it would not be surprising to observe dramatic improvements in the performance of such reversible networks. The efficiency and capability of Neural MJMC profoundly rely on the specific architecture employed, and more sophisticated networks would allow us to deal with systems of increasing complexity. Neural MJMC is a general and transferable method and we can expect it to be applied to a multitude of different systems.

\section{Acknowledgments}

We gratefully acknowledge funding from European Commission (ERC CoG 772230 "ScaleCell"), Deutsche Forschungsgemeinschaft (CRC1114/C03), the MATH + Berlin Mathematics research center (AA1-6), and the International Max Planck Research School IMPRS-CBSC. Furthermore we want to thank Christoph Fröhner, Mohsen Sadeghi and Andreas Mardt for insightful discussions. 
M. S. Albergo, G. Kanwar, and P. E. Shanahan. Flow-based generative models for markov chain monte carlo in lattice field theory. Phys. Rev. D, 100:034515, Aug 2019. doi: 10.1103/PhysRevD.100.034515. URL https://link.aps.org/doi/10.1103/PhysRevD. 100.034515.

Ioan Andricioaei, John E. Straub, and Arthur F. Voter. Smart darting monte carlo. The Journal of Chemical Physics, 114(16):6994-7000, 2001. doi: 10.1063/1.1358861. URL https://doi.org/10.1063/1.1358861.

Tian Qi Chen, Yulia Rubanova, Jesse Bettencourt, and David K Duvenaud. Neural ordinary differential equations. In Advances in neural information processing systems, pages 65716583, 2018.

Yunjie Chen and Benoît Roux. Constant-pH Hybrid Nonequilibrium Molecular Dynamics Monte Carlo Simulation Method. Journal of Chemical Theory and Computation, 11(8): 3919-3931, 2015. doi: 10.1021/acs.jctc.5b00261. URL https://doi.org/10.1021/acs. jctc.5b00261. PMID: 26300709.

Laurent Dinh, Jascha Sohl-Dickstein, and Samy Bengio. Density estimation using Real NVP. arXiv preprint arXiv:1605.08803, 2016.

Charles J Geyer. Markov chain monte carlo maximum likelihood. In Computing Science and Statistics: Proceedings of the 23rd Symposiumon on the Interface, 1991.

Charles J Geyer and Elizabeth A Thompson. Annealing markov chain monte carlo with applications to ancestral inference. Journal of the American Statistical Association, 90 (431):909-920, 1995.

Raza Habib and David Barber. Auxiliary variational MCMC. In International Conference on Learning Representations, 2019. URL https://openreview.net/forum?id=r1N JqsRctX.

W. K. Hastings. Monte Carlo sampling methods using Markov chains and their applications. Biometrika, 57(1):97-109, 1970. ISSN 00063444. URL http://www.jstor.org/stable/ 2334940.

Koji Hukushima and Koji Nemoto. Exchange Monte Carlo method and application to spin glass simulations. Journal of the Physical Society of Japan, 65(6):1604-1608, 1996b.

Diederik P Kingma and Jimmy Ba. Adam: A method for stochastic optimization. arXiv preprint arXiv:1412.6980, 2014.

H. W. Kuhn. The Hungarian method for the assignment problem. Naval Research Logistics Quarterly, 2(12):83-97, 1955. doi: 10.1002/nav.3800020109. URL https: //onlinelibrary.wiley.com/doi/abs/10.1002/nav.3800020109.

Alessandro Laio and Michele Parrinello. Escaping free-energy minima. Proceedings of the National Academy of Sciences, 99(20):12562-12566, 2002. ISSN 0027-8424. doi: 10.1073/ pnas.202427399. URL https://www. pnas.org/content/99/20/12562. 
Daniel Levy, Matt D. Hoffman, and Jascha Sohl-Dickstein. Generalizing hamiltonian monte carlo with neural networks. In International Conference on Learning Representations, 2018. URL https://openreview. net/forum?id=B1n8LexRZ.

E Marinari and G Parisi. Simulated tempering: A new monte carlo scheme. Europhysics Letters (EPL), 19(6):451-458, jul 1992. doi: 10.1209/0295-5075/19/6/002. URL https: //doi.org/10.1209\%2F0295-5075\%2F19\%2F6\%2F002.

N. Metropolis, A.W. Rosenbluth, M.N. Rosenbluth, A.H. Teller, and E. Teller. Equation of State Calculations by Fast Computing Machines. The Journal of Chemical Physics, 21: 1087-1092, June 1953. doi: 10.1063/1.1699114.

Kim A Nicoli, Shinichi Nakajima, Nils Strodthoff, Wojciech Samek, Pan Kessel, et al. Asymptotically Unbiased Generative Neural Sampling. arXiv preprint arXiv:1910.13496, 2019 .

Jerome P. Nilmeier, Gavin E. Crooks, David D. L. Minh, and John D. Chodera. Nonequilibrium candidate monte carlo is an efficient tool for equilibrium simulation. Proceedings of the National Academy of Sciences, 108(45):E1009-E1018, 2011a. ISSN 0027-8424. doi: 10.1073/pnas.1106094108. URL https://www.pnas.org/content/108/45/E1009.

Frank Noé, Simon Olsson, Jonas Köhler, and Hao Wu. Boltzmann generators: Sampling equilibrium states of many-body systems with deep learning. Science, 365(6457), 2019. ISSN 0036-8075. doi: 10.1126/science.aaw1147. URL https://science.sciencemag. org/content/365/6457/eaaw1147.

Danilo Jimenez Rezende and Shakir Mohamed. Variational inference with normalizing flows. arXiv preprint arXiv:1505.05770, 2015.

K. Roberts, R. Sebsebie, and E. Curotto. A rare event sampling method for diffusion Monte Carlo using smart darting. The Journal of Chemical Physics, 136(7):074104, 2012. doi: 10.1063/1.3685453. URL https://doi.org/10.1063/1.3685453.

Huitao Shen, Junwei Liu, and Liang Fu. Self-learning monte carlo with deep neural networks. Phys. Rev. B, 97:205140, May 2018. doi: 10.1103/PhysRevB.97.205140. URL https: //link.aps.org/doi/10.1103/PhysRevB.97.205140.

Jiaming Song, Shengjia Zhao, and Stefano Ermon. A-NICE-MC: Adversarial Training for MCMC. In I. Guyon, U. V. Luxburg, S. Bengio, H. Wallach, R. Fergus, S. Vishwanathan, and R. Garnett, editors, Advances in Neural Information Processing Systems 30, pages 5140-5150. Curran Associates, Inc., 2017. URL http://papers.nips.cc/ paper/7099-a-nice-mc-adversarial-training-for-mcmc.pdf.

Harry A. Stern. Molecular simulation with variable protonation states at constant $\mathrm{pH}$. The Journal of Chemical Physics, 126(16):164112, 2007. doi: 10.1063/1.2731781. URL https://doi.org/10.1063/1.2731781.

Glenn M Torrie and John P Valleau. Nonphysical sampling distributions in monte carlo free-energy estimation: Umbrella sampling. Journal of Computational Physics, 23(2): 187-199, 1977. 
Lionel Walter and Marcus Weber. ConfJump: a fast biomolecular sampling method which drills tunnels through high mountains. Technical Report 06-26, ZIB, Takustr. 7, 14195 Berlin, 2006.

Jun Zhang, Yi Isaac Yang, and Frank Noé. Targeted Adversarial Learning Optimized Sampling. The Journal of Physical Chemistry Letters, 10(19):5791-5797, 2019. doi: 10.1021/acs.jpclett.9b02173. URL https://doi.org/10.1021/acs.jpclett.9b02173. PMID: 31522495. 


\section{Appendix A: Entropy difference}

The (Gibbs) entropy of a system is defined as

$$
S_{X}=-k_{B} \int_{\Omega} p_{X}(x) \log p_{X}(x) d x
$$

For a bijective function $y=\mu(x)$ we can apply the change of variable formula to compute the change in entropy under the transformation. With the transformed density being $p_{Y}(y)=p_{X}\left(\mu^{-1}(y)\right)\left|\operatorname{det} J_{\mu^{-1}}(y)\right|$ we find

$$
\begin{aligned}
S_{X}=-k_{B} \int_{\mu(\Omega)} p_{Y}(y) \log p_{Y}(y)\left|\operatorname{det} J_{\mu=1}(y)\right| d y & \\
& =S_{Y}-k_{B} \int_{\mu(\Omega)} p_{Y}(y) \log \left|\operatorname{det} J_{\mu^{-1}}(y)\right| d y .
\end{aligned}
$$

Thus the difference in entropy under the transformation $\mu(\cdot)$ is given as

$$
\Delta S=S_{Y}-S_{X}=-k_{B} \mathbb{E}_{x \sim p_{\Omega}}\left[\log \left|\operatorname{det} J_{\mu}(x)\right|\right]
$$

where we used the inverse function theorem to compute the Jacobian.

\section{Appendix B: Functional form of potentials}

Here we give the exact functional form of the potentials used to demonstrate the proposed method.

TRIPLE WELL POTENTIAL

Triple well potential is a $2 \mathrm{D}$ potential surface given by

$$
V(\mathbf{x})=\sum_{i}-a_{i} \exp \left[-\left(\mathbf{x}-\mathbf{m}_{i}\right)^{T} \Sigma_{i}\left(\mathbf{x}-\mathbf{m}_{i}\right)\right]+b\|\mathbf{x}\|^{2}
$$

with $b=0.1$ and other parameters given in table 1 .

Table 1: Parameters of the triple well potential

\begin{tabular}{|c|c|c|c|}
\hline $\mathrm{i}$ & $\Sigma_{i}$ & $\mathbf{m}_{i}^{T}$ & $a_{i}$ \\
\hline \hline 1 & $\operatorname{diag}(0.5,0.3)$ & $(-2.2,-1)$ & 5 \\
\hline 2 & $\operatorname{diag}(0.5,0.4)$ & $(0,2)$ & 5 \\
\hline 3 & $\operatorname{diag}(0.4,0.5)$ & $(2,-0.8)$ & 5 \\
\hline
\end{tabular}

\section{Dimer in A LENNARd Jones BATH}

The the dimer system is described in detail in Ref. Noé et al. (2019), and the specific parameters used in this paper are given in table 2 . 
Table 2: Parameters of the particle dimer system

\begin{tabular}{|c|c|c|c|c|c|c|c|c|c|}
\hline Parameter & $\epsilon$ & $\sigma$ & $k_{d}$ & $d_{0}$ & $a$ & $b$ & $c$ & $l_{\text {box }}$ & $k_{\text {box }}$ \\
\hline \hline Value & 1.0 & 1.0 & 20 & 1.5 & 25 & 10 & 0.0 & 3.0 & 100 \\
\hline
\end{tabular}

\section{Appendix C: Details of the network architecture}

The RNVP network consists of may subsequent blocks which are depicted in Fig. 1 (right). Each of these blocks consists of 4 independent networks, two for scaling and two for translation. All networks use leaky ReLU in each hidden layer. The output of the scaling networks uses a hyperbolic tangent scaled by a trainable scalar. The output of the translation networks is linear. Adam Kingma and Ba (2014) is used as optimizer with standard parameters and a learning rate depending on the system. Table 3 gives an overview of the exact network architectures and hyperparameters used in the experiments.

Table 3: Parameters of the RNVP networks used in the experiments

\begin{tabular}{|l|c|c|}
\hline & DW & particles \\
\hline \hline \# blocks & 10 & 20 \\
\hline hidden dimensions & {$[20,20,20]$} & {$[76,76,76]$} \\
\hline \# parameters & $3.6 \times 10^{4}$ & $1.4 \times 10^{6}$ \\
\hline \# training samples per bias and core & $1 \times 10^{5}$ & $1 \times 10^{5}$ \\
\hline bias strengths $/ k_{B} T$ & {$[10,0]$} & {$[500,10,5,2]$} \\
\hline learning rate & $10^{-3}$ & {$\left[10^{-3}, 10^{-4}, 10^{-4}, 10^{-5}\right]$} \\
\hline batchsize & 2000 & 8192 \\
\hline
\end{tabular}

\section{Data and materials}

Computer code to generate the results presented in this paper is available at here. 